\title{
A Study on the Application of the Chinese characters in teaching Chinese as a foreign language
}

\author{
Shou-yan Miao ${ }^{1,2}$ \\ 1) School of Liberal Arts, Linyi University, Linyi ,Shandong, China. \\ ${ }^{2)}$ School of Liberal Arts, Nanjing University, Nanjing, Jiangsu, China.
}

Abstract: Chinese characters teaching have been the weak link of Teaching Chinese as a Foreign Language for a long time. As Chinese visual symbol system, Chinese characters manifest the immense rationality and logicality, which means form of the written character, is fixed and that specific character is used for specific purpose. At the same time, Chinese characters have strong word-formation power. From the perspective of Chinese character itself, it is the organic entity of form, sound and meaning, which is taking form as core, meaning as content and sound as cover. What's more, Chinese character is the carrier of the most fundamental and profound culture. TCFL, if teachers neglect the features of Chinese characters, often makes it hard to study it. Therefore, in order to improve the level of TCFL, teachers should have foothold in features of Chinese characters, be on the basis of font, sum up the pronunciation rules and then analyze the meaning..

Keywords: characteristics of the Chinese characters; Teaching Chinese as a foreign language; Word standard

\section{汉字特点在对外汉语教学中运用研究}

\author{
苗守艳 ${ }^{1,2}$ \\ 1) 临沂大学文学院, 临沂, 山东, 中国 \\ 2) 南京大学文学院, 南京, 江苏, 中国
}

摘 要 汉字教学一直是对外汉语教学的薄弱环节。作为汉语的视觉符号系统, 汉字表现出极强的理性和逻辑性, 形体固定, 专 字专用, 又具有很强的构词能力; 从汉字自身来看, 它是形、音、义的有机统一体, 以形为核心, 以义为内容, 以音为物质外壳; 此外, 汉字还是最基本、最深厚的文化载体。因此, 在对外汉语教学中, 我们要利用汉字本身特点开展汉字教学, 主要以字形为切入点, 归 纳汉字读音规律, 进而分析汉字的意义, 逐步提高对外汉语教学水平。

关键词 汉字特点; 对外汉语教学; 字本位

\section{1. 引言}

对外汉语教学是对母语为非汉语的学习者进行的第二 语言教学, 有助于我们向世界推广汉语, 以增进世界各国 对中国的了解。它不同于第一语言的习得, 无论是否在目 的语环境, 都是在正规的教学程序下所进行的课堂教学。 但一项调查显示, 学习汉语需要的时间相当于学习一门欧 洲语言的三倍, 主要原因主要汉字的难教难学。对外汉语 教学中,我们就需要结合汉字本身特点进行教学.

\section{2. 汉字的特点}

汉字作为意音文字不同于拼音文字，“汉字属于古典文 字阶段, 它们是一个系统的两个阶段”[1]。汉字是唯一没有 消亡的从象形字到形声字、会意字交融相合和不断发展的 文字。在现代社会, 汉字经历了“存废问题”和“拼音化问题” 的波折坎坷。上世纪 90 年代的“字本位”理论概括了汉字特 点, 人们将以字为汉语基本结构单位的汉语研究思路称为 “字本位”，它与以“词”为基本结构单位的语言理论是两种 不同的理论体系。在中国人的观念里, “字”是中心主题, “词”在许多不同的意义上都是辅助性的副题。”[2]这从汉字 
的书写上就能看出来, 每字呈方块形, 不连写, 只见字不 见词。“字”才是汉语结构的本位, 是研究语音、语义、语 法、词汇的交汇点, 更是对外汉语教学不可忽视特点。

\section{1 汉字具有理性和逻辑性}

(1)形体固定的汉字, 专字专用, 具有很大的灵活性和 独立性, 与缺少形态变化的汉语是相适应的。汉语是单音 节语, 它的结构单位本身没有语法的变化。一般说来, 一 个汉字代表一个音节, 一个音节用一个汉字表示, “儿化音” 以及少量多音节汉字, 如“攰” (十克)、“兞” (千克) 除外。 古代汉语的词和语素基本上都是单音节的, 汉字记录汉语 的词或语素, 在大多数情况下汉字、音节、语素和词基本 上是一致的。例如:“巧言令色, 鲜矣仁”(《论语・学而》)。

(2)汉字经历了形体的变化, 却还是表示同一个字。从 古代汉语到现代汉语, 语音面貌发生了很大的变化, 但是 方块汉字没有变, “一个字从管书到隶书, 到楷书, 是书写 形体本身的变化, 还是同一个字”[3]。如“明”字的形体演变, 从甲骨文到楷体发生很大变化, 但仍做一个字。

(3)汉字具有极强的组词造句能力。古代汉语中, 汉字 主要是记录词, 一个字就是一个词。现代汉语的双音词是 在古代汉语单音词的基础上, 根据并列、偏正、主谓等原 则产生的, 据统计常用汉字仅有 6000-7000 个, 但一个汉 字可以组出几个, 甚至几十个词, 说明汉字有很强组词造 句能力。

\section{2 融形、音、义于一体}

“声不能传于异地, 留于异时, 于是乎书之为文字。文 字者, 所以为意与声之迹也。”[3]音、义是语言必备的两种 元素, 而汉字基本都结合了形、音、义三种元素, 以形为 核心, 以意为内容, 以音为物质外壳, 有机地构成了一个 三维合成信息码。从古至今, 汉字的形体、读音和意义都 发生了较大的变化, 但汉字的基本性质没变, 现代汉字仍 然是形、音、义的统一体。

每个独立的汉字, 无论其内部笔画有多少, 结构差异 多大, 都能表示至少一个音节。汉字的字形与语音发生直 接联系始自形声字的产生, 《说文解字》收字 9353 个, 形 声字多达 7697 个, 占了总数的 $82 \%$ 还多, 形声字的出现使 汉字的形体和读音具有了切实的联系, 汉字的形体开始具 有了表音的功能。在现代近 7000 个通用字中, 形声字有 3975 个, 占 $56.7 \%$, 形声字在现代汉字中的地位和作用仍 然相当重要。

汉字造字之初是根据字义构形的, 汉字字形“总是携带 着可供分析的意义信息。”[4]在古代汉字阶段, 字形与字义
的关系主要体现在字形与本义的关系上, 而现代汉字阶段 字形与字义的关系主要是字形与现代常用义的关系。

\section{3 承载着丰富的文化内涵}

汉字具有理性化的特点, 但这决不意味着汉字是一些 互无关联的抽象符号, 或是抽象的、冰冷的描述工具, 汉 字是最基本、最深厚的文化载体。

(1)汉字是古人心理和思维活动的产物。例如, “筷子” 的“筷”就反映了古代的民俗, “筷”, 古代写作“箸”, “箸” 与“住”声音相同, 因船家忌讳“住”, 于是反其意取名为快, 后来又加上了竹字头, 变成了今天的“筷”。

(2)汉字是我们解读史前文化, 记录当代文化, 储存未 来文化的重要工具。蔡邑在《笔赋》中赞扬汉字“书乾坤之 阴阳, 赞三皇之功勋”, 将汉字与宣扬儒家思想和政治理想 联系起来, 赋予其社会政治功能。再如, 许慎的《说文序》 记载: “文字者, 经义之本, 王政之始, 前人所以垂后, 后 人所以识古”, 作为汉语言的记录符号, 汉字还承担着文化 传承的重任。此外, 汉字包含了典型的“东方思维方式”, 即: 具象、隐喻和会意。

(3)新字的产生和旧字的消亡反映出某些文化因素的变 动。古代没有现代化的交通工具, 马是最重要的代脚力, 所以不同毛色不同性别不同特点的马都有自己的专门称呼 和用字，如：千里马叫做“駬”，三四岁的马叫做“駣”，马 高七尺叫“騋”, 马高六尺为“驕”。但是随着时代的发展和 进步, 马的作用几乎完全被现代的各式交通工具给取代了, 因此, 很多从“马”的字都变成了不再流通的死字了。

\section{3. 对外汉语教学中巧用汉字特点}

目前对外汉语教学中采用的汉字教学策略总得来说割 裂了汉字的特点, 而仅从一个或某几个方面讲解汉字, 违 背了汉字整体性的原则, 极易导致汉字越学越难。为推进 对外汉字教学改革, 我们迫切需要将汉字的特点运用到对 外汉字教学中去。

\section{1 以字形为切入点}

汉字是一种记录语言的符号, 属于象形表意体系的文 宇。自从秦朝统一中国后, 幅员辽阔的中国大地, 虽然方 言各不相同, 但是人们却通过汉字增强了民族的认同感。 即使方言不可以沟通, 但是只要有汉字就可以写出来沟通, 汉字是看的, 它具有较强的视觉特征, 世界上没有哪个国 家和民族的文字能像汉字一样, 把书写的过程变成艺术创 作的过程, 把书写的结果变成艺术创作的成果。

对于汉字, 我们以“形”为基点进行研究, 在于更好地 
理解汉字在视觉传达方面所表现出的特征, 利用这一特征 开展对外汉语教学, 利于留学生跨越文字障碍, 了解汉字 的形体演变, 因为比如, “象形者, 画成其物, 随体诘诎, 日月是也。”“山、日、月”这些与自然事物比较相似的字形 大多意义比较生动易懂, 适合讲授给汉字入门阶段的学生, 以增加趣味性。象形是独体字, 只能孤立地、静态地表达 意义, 而会意字和指事字则集合地、动态地表达意义, 比 如, “梦”, 其甲骨文为字形为睡着的人躺在床上, 一只手 指着眼睛, 因此可以分析其字义为“夜间在床上睡觉, 眼前 模糊看不清”, 这就是“梦”。

\section{2 归纳汉字读音规律}

与印欧语系文字不同, 汉字的字音不能通过形体直接 拼读, 但这并不代表汉字读音无规律可寻。谐音与形声现 象是汉字读音的两大特点, 分别代表了汉字读音与字义和 字形的联系。

汉语发音原理有五种情形: 拟音、音拟、谐音、紧缩、 外来。其中拟音、音拟、谐音这三种方式, 无论汉语或其 他什么语言, 其原始的音义联系都是如此产生的。拟音是 模拟事物的声音。如: 动作时发出的声音, 呼、吸、呵、 哈、欠、吼、嚎、号、噮、啰嗦等, 拟音是汉字发音的源 头。音拟是指有些事物并不发音, 人主动用声音来做形象 的表达。如: “圆”, 用圆的唇型模拟圆的形状, 进而表达 圆的意义。

谐音是汉语语音的一个显著的特点。汉语普通话共有 四百多个音节, 即使与“四声”配合, 也只有一千三百多个, 而汉字的总数在五万个以上, 如果以一般字典所收的八千 字为计算对象, 那么, 平均每个音节也有六个同音字。比 如, “hàn”这个音节, 就可以表示“汉、汗、瀚、焊、旱、 憾、感、悍” 等字形。谐音即音近意通。如: 声旁表音兼作 表意, “线、残、或、栈、贱、笺、饯、浅、钱”, 都有少、 小或分割之意。但也有很多虽然意通音近, 但字形却有很 大差别, “团、管、环、卷、旋、玄、圆、园、院、垣、丸、 弯、碗、玩、挽、转、剜、钻”, 几乎都是不同的偏旁。

形声现象是汉语语音的另一特点。形声字是汉字中最 多最常见的一种字, 有首歌谣对形声字概括的非常到位: “形声字, 好识记, 声旁帮着读字音, 形旁帮着辨字义。”[3] 从形旁和声旁在汉字里的分布位置上看, 形声字可以分为 八种: 左形右声, 如“防”; 右形左声, 如“胡”; 上形下声, 如“字”; 下形上声, 如“斧”; 声占一角, 如“旗”; 形占一角, 如“栽”; 外形内声, 如“园”; 内形外声, 如“辩”。方块汉字 不能直接拼读出语音, 但通过建立字音与字形字义的联系, 汉字读音还是有规律可循的。

\section{3 据形析义}

“义起于形, 形为义设, 义为形存”的独特个性使汉字 为人们的认读和理解带来便利。“汉字构形的最大特点是它 要根据所表达的意义来构形, 因此, 汉字的形体总是携带 着可供分析的意义信息。”[5]汉字是一种象形表意文字, 形 体上具有可视性, 主要表现为汉字能够见形知义, 通过考 察汉字字形, 就可以解说该字的字义, 发掘、揭示字中隐 藏的丰富意蕴。

比如“马”本是象形字, 它本身固定下来的“马”这个意 义始终没变, 当它作意符组成“骏、驭、驰、驯、骑”等形 声字时, 也是靠它自身固定下来的意义来表示与“马”有关 的意思。只要了解“马”的意义, 一旦见到以“马”为偏旁的 字就会自然地联想到这个字所代表的词的意义与马有关。

但是, 汉字是不断发展变化的。正如安子介所说, 汉 字是中华文明的基石, 它没有像古代的指南针、造纸术、 印刷术和火药那样成为历史的遗迹, 而是历久常新。例如, “骗”在《集韵》中表示: “跃而乘马也”, “骄”在《说文解字》 中表示: “马高六尺为骄”。字义本与“马”有直接联系, 可 是现代常用义“欺骗”“骄傲”与“马”无关, 所以, 关于汉字的 形义关系, 在对外汉字教学中需要用发展的眼光看待。

\section{4. 结语}

汉字与汉语的学习是相互促进的, 汉字是汉语学习的 基础, 汉语是汉字学习的前提。汉字的特点成为更好学习 汉语桥梁,充分利用汉字特点进行对外汉语教学必经之路.

\section{参考文献(References)}

[1] Jin-ming Zhao. Research On Chinese as a second language learners' language systems. Peking: The Commercial Press, 2006: 2-34.

[2] Yu-lin Yuan, Exploration and Development of Chinese Modern linguistics--Zhao Yuanren's linguistic selection of papers. Peking: Tsinghua University Press, 1992.64.

[3] Fei-sheng Ye, Tong-QiangXu. Linguistics outline. Peking: Peking University Press, 2010.163-184.

[4] LIDa-sui. An Overview of TCSOL Development and Studies. Journal of College of Chinese Language and Culture of Jinan University, 2004,(02).

[5] Jian-xia Ren. For Chinese characters teaching with the characteristics of Chinese language and characters. Educational Practice and Research, 2011 (09). 